\title{
Dietl's Crisis: An Under-Recognized Clinical Entity in the Pediatric Population
}

\author{
Madhu Alagiri, Sapan K. Polepalle \\ Division of Urology, University of California San Diego Medical Center, San Diego, California, \\ USA
}

\begin{abstract}
Objective: To characterize and determine whether patients with recurrent abdominal symptoms and associated ureteropelvic junction obstruction (UPJO) (Dietl's crisis) are effectively treated by pyeloplasty and to determine criteria for evaluating UPJO in childhood abdominal pain.

Materials and Methods: A retrospective chart review from 1998 to 2001 was performed to identify patients with Dietl's crisis and associated UPJO. Chart review included presenting symptoms, location of lesion, condition of the affected renal unit, referral method, and surgery success.

Results: Eight patients (7 male and 1 female) were identified with Dietl's crisis. All eight were initially misdiagnosed and spent at least a year with significant pain symptoms before being properly diagnosed. Only one patient had associated urologic complaints. Renal scan split functions of the affected renal unit ranged from $34 \%$ to $51 \%$. One nephrectomy and seven pyeloplasties were performed and resolution of all patients' abdominal symptoms, including pain, resolved.

Conclusions: Children with Dietl's crisis often suffer a delay in diagnosis; the clinical entity appears to be under-diagnosed. Renal parenchyma is typically preserved, and there is a paucity of associated urologic complaints. Once properly diagnosed, patients are well served by a pyeloplasty. Children with periumbilical pain and vomiting, particularly males, would benefit from ultrasound imaging.
\end{abstract}

Key words: children; abdominal pain; diagnosis; ureteral obstruction; hydronephrosis

Int Braz J Urol. 2006; 32: 451-3

\section{INTRODUCTION}

Recurrent abdominal pain is a relatively common complaint affecting $10 \%$ of school-age children (1). The etiology is often thought to be psychogenic with an underlying organic cause present in less than $10 \%$ of such patients (2). Management focuses on constipation avoidance and identification of relevant psychosocial issues. Routine abdominal imaging is not a common practice. When imaging is performed, obstructing hydronephrosis may be identified and the patient is referred to a pediatric urologist. The pediatric urologist is then faced with the task of determining whether the ureteropelvic junction obstruction (UPJO) is the cause of the symptoms. A review of the literature shows that published reports are sparse regarding this matter. Therefore, a retrospective review was performed to 
characterize patients with Dietl's crisis and to determine whether repair of the UPJO results in resolution of symptoms.

\section{MATERIALS AND METHODS}

A retrospective review of one pediatric urology clinic's patient charts from 1998 to 2001 was performed to identify patients with a history of severe, episodic, periumbilical pain associated with nausea and vomiting and a hydronephrotic kidney. The patient charts were reviewed for preoperative and postoperative symptoms, location of lesion, condition of affected renal unit, associated urologic history, results of urologic imaging studies, and method of referral. Approximately 122 patients were evaluated for UPJO during the period of the study. Of this group, eight patients ( 7 male, 1 female) fit the criteria for Dietl's crisis and were diagnosed with UPJO. The mean age was 11.8 years with a range between 5 and 19 years. The patients are presented in chronological order in Table-1. The preoperative renal scan results for the patients undergoing pyeloplasty are also included in Table-1.

\section{RESULTS}

All patients had a history of recurrent, severe abdominal pain with associated nausea and non-bil- ious vomiting requiring repeated emergency room or office visits. None of the male patients had an associated urologic history. The lone female had a history of a non-febrile urinary tract infection. All 8 patients had symptoms for a minimum of 1 year prior to urologic referral. Patient \#3 had only one functioning kidney. Patient \#4 had a long history of severe abdominal pain thought to be psychogenic in origin. He presented one day to the emergency room with especially severe, incapacitating pain. An emergent CT scan identified a hydronephrotic kidney with extensive intrapelvic and perirenal hemorrhage. Upon surgical exploration, he was found to have extensive hemorrhagic infarction necessitating a nephrectomy. The remaining patients underwent open pyeloplasty.

Postoperative pain assessments noted that all patients had uniform resolution of their abdominal pain, nausea, and vomiting. Follow-up diuretic renal scans confirmed resolution of UPJO.

\section{DISCUSSION}

Dietl's crisis, first reported by Josef Dietl in 1864 , is described as episodic, crampy upper abdominal pain, nausea, and vomiting associated with intermittent renal pelvic obstruction (3). In 1987, Flotte reported the problem in an 11-year-old child who had monthly episodic pain for half a year before she was diagnosed with a UPJO (4). After surgical correction, her abdominal complaints ceased. Ward and Brereton

Table 1 - Patients with left $(L)$ or right $(R)$ ureteropelvic junction obstruction (UPJO) and abdominal pain presented in chronological order.

\begin{tabular}{lcccccl}
\hline \multirow{2}{*}{ Patient } & Sex & Age (years) & Diagnosis & Seft (\%) & Right (\%) & Procedure \\
\hline 1 & M & 15 & L UPJO & 51 & 49 & L Pyeloplasty \\
2 & F & 6 & L UPJO & 51 & 49 & L Pyeloplasty \\
3 & M & 19 & R UPJO & 0 & 100 & R Pyeloplasty \\
4 & M & 16 & L UPJO & N/A & N/A & L Nephrectomy \\
5 & M & 10 & L UPJO & 46 & 54 & L Pyeloplasty \\
6 & M & 5 & L UPJO & 66 & 34 & L Pyeloplasty \\
7 & 14 & R UPJO & 64 & 36 & R Pyeloplasty \\
8 & M & 14 & R UPJO & 63 & 37 & R Pyeloplasty \\
\hline
\end{tabular}


presented 3 boys with recurrent abdominal pain and UPJO (5). All 3 children had abdominal pain and bouts of non-bilious vomiting for several years before the diagnosis of UPJO was made. The authors concluded that the evaluation of episodic abdominal pain and non-bilious vomiting should include an abdominal ultrasound to exclude UPJO. Belman presented a case of a 6-year-old boy with a 1-year history of frequent, severe, episodic abdominal pain associated to nausea and vomiting requiring pyeloplasty (6).

Complaints of abdominal pain are common in school-age children. The complaint is present in approximately $10 \%$ of all school-age children, and is found to have an organic etiology in fewer than $10 \%$ of this group (2). The primary etiologies are thought to be psychosocial and gastrointestinally related. In a review of the charts of 65 children and adolescents who had ultrasonography for recurrent abdominal pain, Shanon et al. (7) found that $81 \%$ had normal results and $19 \%$ had abnormal results. In no case could the abdominal pain be attributed to the abnormal finding. O'Donnell documented that only one of 32 children with UPJO reported recurrent abdominal pain, and UPJO was the cause in only $1 \%$ of cases in Apley's review of abdominal pain in children $(1,8)$.

The most striking finding in the case reports and in the present series is the delay in diagnosis. The most likely reason for a delay in diagnosis and management is the well-supported belief that abdominal imaging studies are of limited use in children who have abdominal pain. Nevertheless, an argument could be made for urologic imaging in a child, particularly a male, who has prolonged, episodic abdominal pain and vomiting. Despite its apparent rarity, we identified Dietl's crisis in eight patients in one pediatric urologist's clinic over a 3-year period. This finding would suggest that this is an under-recognized clinical entity whose true prevalence must be determined.

\section{CONCLUSION}

Dietl's crisis is not a well-recognized clinical entity, which leads to delay in proper diagnosis. Patients are predominantly male with good preservation of the affected renal unit. The absence of associ- ated urologic symptoms contributes to the late referral to a pediatric urologist. Once the proper diagnosis is made, patients are well served by a pyeloplasty with resolution of pain symptoms.

\section{CONFLICT OF INTEREST}

None declared.

\section{REFERENCES}

1. Apley J: The Child with Abdominal Pains. 2nd ed., Oxford, Blackwell Scientific Publications. 1975.

2. McGrath PJ, Goodman JT, Firestone P, Shipman R, Peters S: Recurrent abdominal pain: a psychogenic disorder? Arch Dis Child. 1983;58: 888-90.

3. Dietl J: Wandernde nieren and deren einklemmung. Wien Med Wochenschr. 1864; 14: 153-66.

4. Flotte TR: Dietl syndrome: intermittent ureteropelvic junction obstruction as a cause of episodic abdominal pain. Pediatrics. 1988; 82: 792-4.

5. Ward HC, Brereton RJ: Pelviureteric junction obstruction and recurrent abdominal pain in childhood. Br J Surg. 1989; 76: 818-9.

6. Belman AB: Ureteropelvic junction obstruction as a cause for intermittent abdominal pain in children. Pediatrics. 1991; 88: 1066-9.

7. Shanon A, Martin DJ, Feldman W: Ultrasonographic studies in the management of recurrent abdominal pain. Pediatrics. 1990; 86: 35-8.

8. O'Donnell B: Abdominal Pain in Childhood. Oxford, Blackwell Scientific Publications. 1985.

Accepted after revision:

June 10, 2006 